\title{
An approach to the Internet of Things system with nomadic units developing
}

\author{
Larysa S. Globa, Ivan Ishchenko, Nataliia Gvozdetska, Andrii Zakharchuk, Oleh Zvonarov \\ National Technical University of Ukraine - Kyiv Polytechnic Institutel, Institute of Telecommunications \\ Kyiv, Ukraine \\ e-mail: lgloba@its.kpi.ua,n.gvozdetska@gmail.com
}

\begin{abstract}
The Internet-of-Things (IoT) is penetrating in every sphere of a human life simplifying his everyday life and rising his labor efficiency. There are a lot of challenges on its way of development. Additional challenges appear when considering the IoT systems with nomadic units. The paper is dedicated to the problem of data collecting, processing and storing in the IoT system containing nomadic units. One of the difficulties in such systems' developing is a problem of data volumes reducing for its transferring. For system efficiency improving it is proposed to use an additional transitional hub interacting with the Cloud services.
\end{abstract}

Keywords-Internet of Things; nomadic units; data collecting, processing, storing; Azure IoT Hub; devices interaction

\section{INTRODUCTION}

The Internet of Things (IoT) conception is quite new but in the same time it is very fast developing. The sense of this conception is that all the devices used by human are interconnected and smart. It means that those things, which have already helped us for many years, get an opportunity now to operate more independently due to the self-regulation and communication with other devices and systems. There is not a unique definition for the Internet of Things (IoT), but most of them agree that it is composed of devices capable of sensing/actuation, communication and processing [3]

The IoT is expected to have great impact on several aspects of our everyday-life, enabling the development of a wide range of applications, including: sensor data aggregation, intelligent transportation schemes, business management, environmental monitoring, e-health and many others [1].

The IoT meets a lot of challenges on its way of developing. The most notable are the question of data security providing, constant availability requirements, data storage organizing, server technologies efficiency and others. Many technologies aimed to deal with these challenges are being invented every year all over the world, but it causes a great diversity of all IoT systems and, as a result, some problems with their integration. The problems of interaction of different units in such systems are especially performed in those systems containing nomadic (mobile) units, taking into account that several heterogeneous technologies need to be used.

This paper is dedicated to the technologies of data collecting and processing with a help of Azure IoT Hub Cloud service and of data storage selecting rules in the IoT systems containing nomadic units. Also in this paper existing technologies and protocols are analyzed and compared, and the most appropriate way of devices interconnection and entire system structure building is proposed. The advantages and disadvantages of this solution are substantiated by using an example of the real IoT system.

There are a lot of solutions for IoT systems containing only static units. The structure of these systems is defined and is shown on the figure 1 .

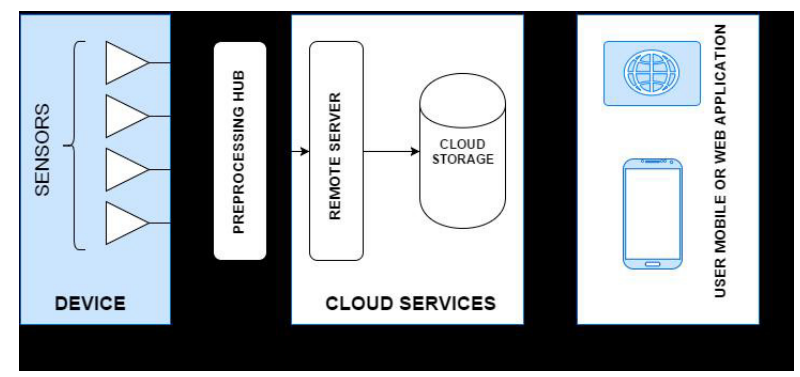

Fig. 1. - General structure of the typical IoT system\|

Being the telecom system, the IoT system is possible to be bound to the OSI model. It is necessary to pay attention to every layer between all the main components of the system:

1. board-to-board connection within a device;

2. device-to-preprocessing hub connection;

3. preprocessing hub-to-cloud connection; 
4. cloud service-with-user application communication;

5. remote server giving commands to device.

For systems with nomadic units have been also proposed some decisions. One of them is new 3GPP Standard for IoT - a special technology to address the requirements of the IoT. The new technology will provide improved indoor coverage, support of a massive number of low-throughput devices, low delay sensitivity, ultralow device cost, low device power consumption, and optimized network architecture [6].

The technology, described above, has a great potential of development, but this paper is aimed to find a solution based on usage of more common technologies for narrow range of systems. For such kind of system implementation there is no common developed infrastructure yet. To make a decision universal it is a need to use existing infrastructure.

In case of an existing infrastructure using the requirements are: high availability of any unit (including mobile one) everywhere, an efficient interaction between these units, reducing of data volumes being transferred between the device and cloud and vice versa. The process of data volumes reducing is very important and it is a need to transfer only those data that contains a sense for end user.

So, there is an actual problem of improving an existing architecture in direction to prove interaction between nomadic units using common technologies.

\section{APPROACH TO THE PROBLEMS SOLUTION}

The mobility of these nomadic units can be considered in different areas of application. Talking about the Smart House conception, mobility is presented only within the home. It doesn't cause any essential problems because a majority of the modern houses are provided with some kind of network (usually it can be IEEE 802.11 (Wi-Fi) network or IEEE 802.15 (Bluetooth) network). But these technologies are not suitable for a number of IoT systems that require their units' interconnection at large distances.

According to this statement it is suggested to modify architecture to provide a connectivity of standalone nomadic unit using existing mobile networks. The structure of such system is shown on the figure 2 .

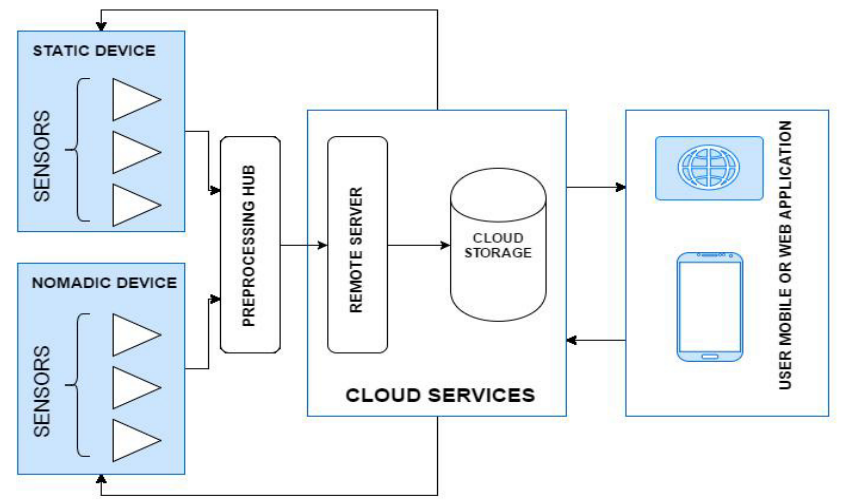

Fig. 2. - General structure of the IoT system including nomadic units

The protocols of the network and transport layer of the OSI model for both variants of system configuration are quite definitely specified. Being an Internet using system, IoT uses TCP/IP stack of protocols on these layers. But there are some problems with an application and physical layers as the endpoints of the IoT system are smart devices.

Another problem is caused by the lack of the efficient data storing and processing mechanisms. Being a complicated solution itself, IoT system requires a huge computing powers and storage with as large capabilities as possible. Due to these requirements Cloud technologies are mostly used.

For a system with nomadic units it is necessary to implement:

1. to provide constant stable connection of the nomadic unit and static preprocessing hub;

2. to keep time synchronization between these parts of the system and how to manage data transfer efficiently;

3. to choose technologies and protocols are possible to use in such system.

4. to arrange data storing and processing in the Cloud.

The decisions proposed in this paper are possible to use in a wide range of devices, which general conception of a structure requires static and nomadic (mobile) units interconnection. 


\section{PROPOSED ARCHITECTURE FOR SYSTEMS WITH NOMADIC UNITS}

According to the general structure, an architecture including such specific units as IoT Hub, Stream Analytics and Azure storage services, which are described below, is shown on the figure 3 .

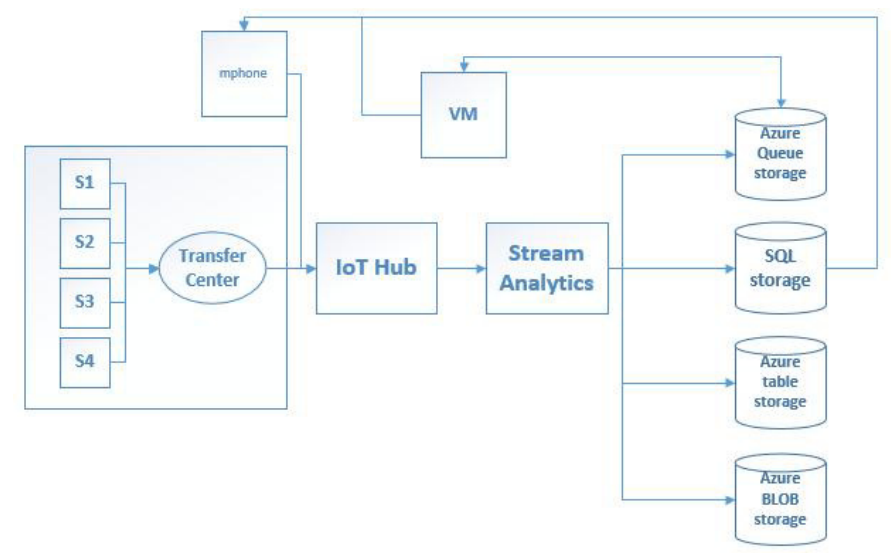

Fig. 3. The architecture of the proposed system

This architecture is used to provide:

1. Correct interaction between physical units of the system;

2. Transparency of Device-to-Cloud and Cloud-to-Device interaction;

3. Efficient data processing and storing organization.

On the figure 3 are showed such items:

1. S1, S2, S3, S4 - sensors.

2. Transfer Center - a hub (physical device), that arranges data from device to IoT Hub transferring.

3. IoT Hub - gives an opportunity to exchange messages between devices and Cloud services. It is also can be performed as a gateway between Cloud services of the IoT Suite.

4. Azure Stream Analytics - is a module of events processing aimed to provide their fast analysis. It is a service providing telemetry data processing, its aggregation and events revealing.

5. To save and prepare data to be analyzed such types of storage services as BLOB-objects service, Queue-, SQL-, table-storage are used.

6. The features of data visualization and charts design are provided by Azure webapplications and Microsoft Power BI service that are developed and built in configured virtual machine in a Cloud.

\section{A. Physical layer technologies}

Talking about the nomadic unit containing systems it is necessary to define what kind of mobility this system requires. In case of some Smart House module, it is a need to organize constant network availability for the IoT device within the home. Usually it doesn't cause any problems, because a conception of a Smart House involves the stable Internet connection within the whole house provided. In this case the most suitable solutions are IEEE 802.11 Wi-Fi technology using or connect the devices via Bluetooth IEEE 802.15 technology.

The problem appears when much more mobile IoT devices are discussed. In this case it is necessary to provide a stable connection wherever the nomadic unit is. Unfortunately, there is not such common network that covers the whole area of the Earth provided. Only satellite Internet access can be considered as an example of such networks. But the satellite based Internet connection is not economically viable for the IoT systems because of its high prices. IEEE 802.11 Wi-Fi and IEEE 802.15 Bluetooth technologies are not suitable to use as their coverage area is extremely small. The best choice in today's situation is to use mobile GPRS, CDMA, HSPA (for 3G-networks), LTE or other like standards.

In case of necessity of a big amount of data transferring (eg. real-time video) the GPRS technology can be helpless because of its rate. But the typical IoT system does not have any special transfer rate requirements. The data which is typically transferred is a couple of parameters definitions taken from the sensors. It means that the GPRS capabilities are completely enough for such systems. The infrastructure all over the world is quite developed to make a usage of this technology possible.

The advantages of the GPRS using in the IoT systems with the nomadic units:

1. Sufficient rates;

2. Developed infrastructure;

3. Density and common use;

4. Economic efficiency.

The disadvantages of the GPRS using in such systems:

1. Not enough capabilities in case of a big amount of data transferring;

2. Obsolescent technology in comparison with LTE and the others. 


\section{B. Device-to-Cloud and Cloud-to-Device interaction ways}

The IoT system is a specific telecommunication system due to the fact that the endpoints of a communication are devices themselves, not a human (according to the typical scenario). It causes a necessity to organize a specific way of an interaction of the different system units.

The most widely used in the IoT systems protocols are AMQP (Advanced Message Queuing Protocol), MQTT (Message Queue Telemetry Transport) and HTTP or HTTPS (HyperText Transfer Protocol Secure).

Being the most universal, HTTP is widely used in the IoT systems, but it uses its potential rather in the deviceto-cloud interaction than the nomadic-to-static units' connection. Its advantages are simplicity, universality and security (talking about the HTTPS). But it is not really adapted for the IoT systems.

In comparison to the HTTP/HTTPS, AMQP is used in the IoT systems rather due to its reliability and interoperability than the universality. Everyone can easily speak HTTP, but it takes special effort to speak AMQP. AMQP was designed as a protocol including reliable queuing, topic-based publish-and-subscribe messaging, flexible routing, transactions, and security. AMQP exchanges route messages directly-in fanout form, by topic, and also based on headers [5]. The disadvantage of AMQP is that it is suitable mostly for the control plane or server-based analysis functions. It is good to be used between the device and cloud to provide efficient way of queues organizing and reliable messaging, as AMQP is aimed to avoid any losses of messages.

The most adapted to the IoT features is MQTT protocol. As its name states, its main purpose is telemetry, or remote monitoring.

In the proposed solution the MQTT protocol is used because being developed especially for IoT it matches all of the requirements to the IoT system units' proper interaction.

\section{Data storing and processing solution}

To store text-type data or binary data the BLOBobjects storage is used.

Table storage is a storage of keys and attributes. This type of storage is fast accessing and economically efficient.

The telemetry-data from sensors is transferred to the IoT Hub service input with the help of Raspberry Pi microcomputer. This data is analyzed, is divided to separate streams, is checked to be saved from any anomaly data and is written to the different types of storages described above.

For better understanding the sense of the data the Power BI service is used. To form graphics, data is stored in the table storage. The disadvantages of such approach, such as a need of a strong data searching mechanism, are compensated by the high computing power of Azure.

\section{IoT Hub service overview}

Microsoft Azure IoT Hub has its advantages in comparison with earlier IoT services, such as Eventhub and service bus. It is able to receive messages from device to cloud (Telemetry messages, feedback), as well as sending messages and commands from devices to cloud services. It allows user to manage, monitor and connect the huge number of devices supporting HTTP, AMQP and MQTT protocols. The IoT Hub SDK is available for multiple platforms such as Windows, Linux and some RTOS and supports multiple languages such as C, .NET, Java and Node.js.

According to the statements above it is proposed to include such unit as IoT Hub in the typical IoT system containing nomadic units that is described below. IoT Hub is aimed to simplify and rearrange the structure of the typical IoT-system by such functions outgoing: routing, cross-proxy, cache and sources catalogue.

Azure IoT Hub is aimed to provide an easy Devicesto-Cloud access and also arranges a bidirectional connection (Device-to-Cloud and Cloud-to-Device). It performs a full security connection that means that the data will be checked precisely and only after authentication check passing will be stored in a Cloud.

The main thing that differs IoT Hub from the other services is its computing power that allows million queries to be processed just in one second. The information about events processed in the IoT Hub is stored during a week that provides an extra item to the security problem solving.

\section{AN EXAMPLE OF THE REAL IOT SYSTEM CONTAINING NOMADIC UNITS}

As an example of a nomadic unit containing system it is proposed to consider a Smart dog-collar device. This device is aimed to monitor a pet's health and its location.

The usage of this device implies a static preprocessing hub which is constantly placed at home, and a compact collar built-in system which includes a number of sensors 
as a temperature and heart-beat sensors and GPS location defining device.

This collar built-in system can be performed as a nomadic unit of the IoT system and, as a result, all technologies described above can be used in it.

The data collected from the sensors is transferred to the IoT Hub where it is preprocessed and aggregated. An additional aggregation and data volumes reducing is provided by using Stream Analytics service. Than the data is distributed between the Azure storage services according to its type and volume.

The project provides health state of a pet monitor. The telemetry data, that is stored, need to be renovated 2-3 times per day. The results of health parameters measurements are better understanding being visualized with the help of the Power BI service. It is used in project to allow user to see statistical data in the most convenient way.

\section{SUMMARY}

1. In this paper is suggested the approach to the IoT systems containing nomadic units design. This approach allows to use existing technologies efficiently;

2. The aspects of physical layer interaction (concerning mobile unit existing in a system) were researched. The way of interaction using existing mobile networks was proposed;

3. Device-to-Cloud and Cloud-to-Device communication ways are investigated and the most appropriate protocols were chosen;

4. Such system's ability of permanent access was checked on example of a real IoT system (Smart dogcollar device). The advantages and disadvantages of every particular solution are discovered and analyzed.

The future research is aimed to study more deeply such important parameters as availability, reliability and performance in systems with large volumes of data transmission. It is necessary to provide quality evaluation of the functioning of these systems, analysis and optimization of the large volumes of information transfer, as well as the probability of message loss. Another point of future work is connected with studying of system performance and influence of data streams type.

\section{REFERENCES}

[1] Michalis Giannikos, Korina Kokoli, Nikos Fotiou, Giannis F. Marias and George C. Polyzos -Towards Secure and Context-Aware Information Lookup for the Internet of Things\| International Conference on Computing, Networking and Communications, Communications and Information Security Symposium, 2013

[2] Soumya Kanti Datta, Rui Pedro Ferreira Da Costa, Christian Bonnet — Resource Discovery in Internet of Things: Current Trends and Future Standardization Aspects\| IEEE 2015

[3] R. Want, B. N. Schilit, and S. Jenson -Enabling the internet of things\| Computer, vol. 48, no. 1, pp. 2835, Jan 2015.

[4] Hongki Cha, Wonsuk Lee, Jonghong Jeon -Standardization strategy for the Internet of wearable things $\|$, Republic of Korea, ICTC 2015

[5] Jorge E. Luzuriaga , Miguel Perez, Pablo Boronat, Juan Carlos Cano, Carlos Calafate , Pietro Manzoni - A comparative evaluation of AMQP and MQTT protocols over unstable and mobile networks\|, IEEE 12th Consumer Communications and Networking Conference, 2015

[6] Javier Gozalvez -New 3GPP Standard for IoT IEEE - vehicular technology magazine, Mobile radio, IMarch 2016

[7] Roberto Verdone, - Internet of Things: How to Break the Barriers for Business Developmentll, 3th

International Black Sea Conference on Communications and Networking, 2015 